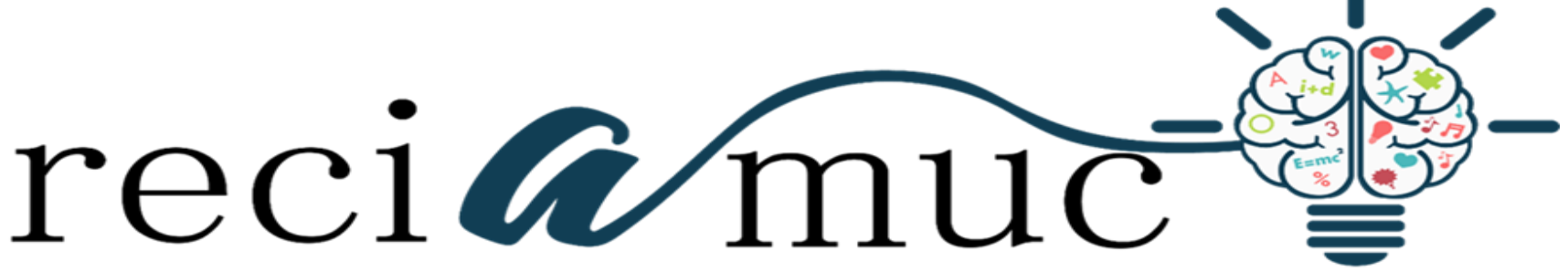

Revista científica de investigación actualización del mundo de las ciencias

Jean Carlos Delgado Macías a; Vilma Geovanna Lara Fajardo b; Luiz Carlos

Flores Tacle ${ }^{\text {c; }}$ Bairon Andrés Sabando Farías ${ }^{\text {d }}$; Edwin Gabriel Aguilar Sánchez ${ }^{\mathrm{e}}$;

Glenda Aracely Fernández Zambrano ${ }^{\mathrm{f}}$

Patologías Específicas de Importancia en la U.C.I

Specific Diseases of Importance in the U.C.I

Revista Científica de Investigación actualización del mundo de las Ciencias. Vol.

3 núm., 2, abril, ISSN: 2588-0748, 2018, pp. 665-687

DOI: $10.26820 / \mathrm{reciamuc/3.(2).abril.2019.665-687}$

URL: $\underline{\text { http://reciamuc.com/index.php/RECIAMUC/article/view/360 }}$

Código UNESCO: 3205 Medicina Interna

Tipo de Investigación: Artículo de Revisión

(C) RECIAMUC; Editorial Saberes del Conocimiento, 2019

Recibido: 15/01/2019

Aceptado: 07/02/2019

Publicado: 01/04/2019

Correspondencia: jancardel_92@outlook.com

a. Médico Cirujano; Médico General Asistencial - Hospital Gustavo Domínguez; Santo Domingo, Ecuador; jancardel192@outlook.com;

b. Médico Residente del Área de Emergencias - Hospital IESS; Ibarra, Ecuador; geova lara@ @otmail.es

c. Doctor en Medicina; Médico Residente del Área de Emergencias - Hospital IESS; Ibarra, Ecuador; lucasfolclorico@gmail.es

d. Médico Cirujano; Médico Residente del Servicio de Cirugía General - Hospital del Norte de Guayaquil IESS Los Ceibos; Guayaquil, Ecuador; bsabando_30322@ hotmail.com

e. Doctor en Medicina; Médico Residente del Área de Anestesiología - Hospital Rodríguez Zambrano; Manta, Ecuador; edwingabrielaguilarsanchez@yahoo.es

f. Doctor en Medicina; Médico Residente del Área de Emergencia - Hospital Rodríguez Zambrano; Manta, Ecuador; glenda_28@hotmail.com 


\section{Patologías Específicas de Importancia en la U.C.I}

Vol. 3, núm. 2., (2019)

Jean Carlos Delgado Macías; Vilma Geovanna Lara Fajardo; Luiz Carlos Flores Tacle; Bairon

Andrés Sabando Farías; Edwin Gabriel Aguilar Sánchez; Glenda Aracely Fernández Zambrano

\section{RESUMEN}

En los últimos años, las UCI han experimentado avances inimaginables en el campo científicotecnológico, los cuales las han convertido en servicios eficientes e imprescindibles para la práctica médica actual. Hoy día, estos servicios tienen una gran relevancia en la epidemiología clínica, así como en las funciones burocrático-administrativas. La UCI, forma parte del último eslabón dentro de un sistema integral de atención al paciente que presenta lesiones o un daño traumático que amenazan la vida y por tanto tienen una alta probabilidad de morir. El objetivo principal de estas salas de atención intensiva es el diagnóstico y tratamiento de los enfermos en situación crítica, es decir enfermos con complicaciones o estados de salud crítica, o de aquellos que comprendan riesgos actuales o potenciales de sufrir complicaciones que pongan en peligro su vida y que el proceso que padece sea potencialmente reversible. Estas unidades de atención especializada médica se interesan por pacientes que se encuentran al límite de sus posibilidades de vida, la ayuda que ofrecen es posible, debido al avance de su tecnología aplicada, encargada de salvar vidas y, en determinadas ocasiones de facilitar que ciertos pacientes permanezcan vivos gracias a máquinas complejas que no hacen más que retrasar su muerte. Entre las patologías más frecuentes y de mayor importancia que son atendidas en estas áreas de salud se encuentran los traumas craneoencefálicos, los EPOC, definidos como una de las enfermedades más comunes de los pulmones que causa dificultad para respirar y sus posibles complicaciones, Cuadros cardiovasculares, Edemas Pulmonares, Traumas de Tórax, Pancreatitis, Insuficiencia Renal Aguda, Infecciones adquiridas en la UCI (generalmente de pulmón), Patologías neuromusculares, Pacientes Sépticos, Pacientes Traumáticos, entre otros cuadros clínicos en situación crítica.

Palabras Claves: Patologías críticas; Unidad de Cuidados Intensivos UCI; Epidemiología Clínica; Complicaciones Médicas; Tecnología Aplicada. 


\title{
Patologías Específicas de Importancia en la U.C.I
}

Vol. 3, núm. 2., (2019)

Jean Carlos Delgado Macías; Vilma Geovanna Lara Fajardo; Luiz Carlos Flores Tacle; Bairon Andrés Sabando Farías; Edwin Gabriel Aguilar Sánchez; Glenda Aracely Fernández Zambrano

\begin{abstract}
In recent years, the UCI have experienced unimaginable advances in the science and technology field, which have become efficient and essential services for the current medical practice. Today, these services have a great relevance in clinical epidemiology, as well as the bureaucratic functions. The UCI, is part of the last link within a comprehensive system of care to the patient who presents a traumatic damage that threaten life and therefore have a high probability of death or injury. The main purpose of these intensive care rooms is the diagnosis and treatment of patients in a critical situation, in other words sick with complications or States of critical health, or those involving actual or potential risk for complications that would endanger his life and that the process that is potentially reversible.These specialized medical care units are interested in patients who are at the limit of its possibilities of life, support offering is possible, due to the advancement of their technology applied, responsible for saving lives, and sometimes of provide that certain patients remain alive by complex machines that do more than delay his death. Among the most frequent pathologies and of greater importance that are served in these areas of health are cranioencephalic trauma, COPD, defined as one of the most common diseases of the lungs that causes difficulty breathing and their possible complications, cardiovascular pictures, pulmonary edema, chest trauma, Pancreatitis, Acute Renal failure, infections acquired in the ICU (usually of lung), neuromuscular diseases, septic patients, trauma patients, between other clinical pictures in critical situation
\end{abstract}

Key Words: Critical pathologies; Unit ICU intensive care; Clinical epidemiology; Medical complications; Applied technology. 


\section{Patologías Específicas de Importancia en la U.C.I}

Vol. 3, núm. 2., (2019)

Jean Carlos Delgado Macías; Vilma Geovanna Lara Fajardo; Luiz Carlos Flores Tacle; Bairon

Andrés Sabando Farías; Edwin Gabriel Aguilar Sánchez; Glenda Aracely Fernández Zambrano

\section{Introducción.}

La Unidad de Cuidados Intensivos UCI, representa para todos los que conviven en ella un espacio simbólico de actuación. Es en esta área geográfica tan cargada de subjetividad donde el médico necesita la objetivación para poder actuar correctamente, pero también la necesita el enfermo: espera ser al mismo tiempo objeto de la atención científica y, a la vez, comprendido y respetado como sujeto (Beoggi, 2015). En otras palabras, estas áreas de servicio ofrecen asistencia de soporte vital al paciente críticamente enfermo como, por ejemplo; ventilación mecánica, terapias de remplazo, monitorización constante, a través de procedimiento invasivos ó no invasivos para la vigilancia de constantes vitales, lo cual facilita la terapéutica a seguir y el beneficio del paciente, evitando el mínimo de complicaciones.

Cabe mencionar que los procedimientos médicos llevados a cabo en la Unidad de Cuidados Intensivos UCI, requiere de la aplicación de una serie de normas de bioseguridad establecidas a nivel mundial, como son la universalidad, uso de método de barrera y la eliminación de desechos. Cabe destacar que, en esta área de atención médica para pacientes en condiciones de salud crítica, no sólo esos realizan procedimientos invasivos, también se lleva un seguimiento microbiológico del paciente, a través de toma de muestra para cultivos como lo son: cultivo de catéter de vía central y secreción endo -traqueal entre los más comunes.

El paciente en condiciones de salud crítica qué requiere ser ingresado en esta Unidad de Cuidados Intensivos UCI, se encuentra asociado de manera general a patologías originadas por accidentes de tránsitos, caídas de altura, arrollamientos, accidente automotor, cuadros cardiovasculares, enfermedades cerebrovasculares, entre otras causas de igual impacto médico, las cuales representan causa de muerte en el mundo. Este tipo de pacientes generalmente son ingresados en el área de emergencia, en donde son asistidos por especialistas, quienes realizan las maniobras adecuadas, para posteriormente ser trasladados a la Unidad de Cuidados Intensivos donde es sometido a procedimientos invasivos, haciéndolo propensos a infecciones nosocomiales. 


\section{Patologías Específicas de Importancia en la U.C.I}

Vol. 3, núm. 2., (2019)

Jean Carlos Delgado Macías; Vilma Geovanna Lara Fajardo; Luiz Carlos Flores Tacle; Bairon Andrés Sabando Farías; Edwin Gabriel Aguilar Sánchez; Glenda Aracely Fernández Zambrano

Los pacientes que son ingresados a la Unidad de Cuidados Intensivos son sometidos a procedimientos invasivos, lo que los lleva a ser propensos de contraer infecciones nosocomiales. En referencia a esto las estadísticas a nivel internacional emanadas por la Organización Mundial de la Salud. (OMS, 2017), revelan que existe "un porcentaje considerable en relación a las infecciones nosocomiales causadas por métodos invasivos donde ocupa el primer lugar los accesos venoso central en las Unidades de Cuidados Intensivos, con un aumento considerable de estas tasas epidemiológicas cada año”. (p. 14). Asimismo, (Hendrick, 2017), refiere que; “en Estados Unidos EEUU se estima que cada año se colocan 3 millones de catéter venoso central, entre los cuales 50.000 y 120.000 pacientes adquieren una bacteriemia relacionada con el catéter de vía central anualmente" (p.5).

Por otra parte (Cols, 2016), realizaron un estudio titulado Epidemiología de las infecciones intrahospitalarias por el uso de catéteres venosos centrales, donde las estadísticas reveladas fueron "el $(41,90 \%)$ de pacientes, quienes presentaron algún tipo de infección intrahospitalaria, seguida de la infección del punto de entrada del catéter de vía central con (30,80\%)" (p.25). El estudio también revelo "que los microorganismos más frecuentes son bacterias Gram positivas, predominando StaphylococcusAureus y Estafilococos coagulasa negativo, siendo el servicio médico con mayor incidencia de casos fue Unidad de Cuidados Intensivos".

Sobre las bases de las consideraciones anteriores, es importante resaltar que la Unidad de Cuidado Intensivo UCI, comprendida en cada Unidad de Asistencia Médica debe contar con los equipos médicos necesarios y tecnología actualizada, la cual le permita cumplir a cabalidad con la atención médica que requiere cada paciente en situación crítica que es ingresado a la misma. Cada profesional debe brindar su aporte desde su particular área de conocimiento y experiencia clínica, a la mejoría global de cada paciente. Esta área de salud integral admite pacientes tanto pediátricos como adultos, con diversas patologías. Este compromiso es verdaderamente grande, dado que representa un área de salud que involucra cuidados y atención exclusiva para cada caso en especial. 


\section{Patologías Específicas de Importancia en la U.C.I}

Vol. 3, núm. 2., (2019)

Jean Carlos Delgado Macías; Vilma Geovanna Lara Fajardo; Luiz Carlos Flores Tacle; Bairon Andrés Sabando Farías; Edwin Gabriel Aguilar Sánchez; Glenda Aracely Fernández Zambrano

En el manejo actual de los pacientes ingresados en la Unidad de cuidados Intensivos UCI, participan médicos de diversas especialidades, enfermeras, kinesiólogos, farmacéuticos clínicos, entre otros. La medicina presente en los conocimientos comprendidos por cada especialista aporta valoraciones científicas y la visión humanista necesaria para la atención de pacientes severamente enfermos, lo que facilita la implementación de herramientas, control y tratamiento de primer orden que logran corregir la enfermedad que traen los casos asistidos en la unidad de pacientes críticos, lográndose con ello que, el trabajo realizado por de equipos multidisciplinarios resulte fundamental, para la toma de decisiones adecuadas y oportunas en pacientes graves, balanceando los riesgos y beneficios de las distintas alternativas terapéuticas existentes.

La mayoría de los escenarios clínicos que ameritan ingresos en la Unidad de Cuidados Intensivos UCI, son representados por cuadros cardiovasculares, cerebrovasculares, sepsis, trauma, Edemas Pulmonares, Traumas de Tórax, Pancreatitis, Insuficiencia Renal Aguda, Infecciones adquiridas en la UCI (generalmente de pulmón), Patologías neuromusculares, Pacientes Sépticos, Pacientes Traumáticos, entre otras patologías en situación grave o crónica; todos ellos, de igual importancia para el especialista intensivista y personal especializado quienes brindan atención médica las 48 horas del día, con el fin de sacar al paciente del estado crítico que presentan.

\section{Tipo de Investigación}

Dentro de toda práctica investigativa, se precisan acciones de carácter metodológico mediante las cuales, se logra conocer y proyectar los eventos posibles que la determinan, así como las características que hacen del acto científico un proceso interactivo ajustado a una realidad posible de ser interpretada. En este sentido, se puede decir, que la presente investigación corresponde al tipo documental, definido por (Dávila, 2012), "se ocupa del estudio de problemas planteados a nivel teórico, la información requerida para abordarlos se encuentra básicamente en materiales impresos, audiovisuales y /o electrónicos". (p.41).

En consideración a esta definición, la orientación metodológica permitió la oportunidad de cumplir con una serie de actividades inherentes a la revisión y lectura de diversos documentos donde se encontraron ideas explicitas relacionadas con los tópicos encargados de identificar a 


\section{Patologías Específicas de Importancia en la U.C.I}

Vol. 3, núm. 2., (2019)

Jean Carlos Delgado Macías; Vilma Geovanna Lara Fajardo; Luiz Carlos Flores Tacle; Bairon

Andrés Sabando Farías; Edwin Gabriel Aguilar Sánchez; Glenda Aracely Fernández Zambrano

cada característica insertada en el estudio. Por lo tanto, se realizaron continuas interpretaciones con el claro propósito de revisar aquellas apreciaciones o investigaciones propuestas por diferentes investigadores, para luego dar la respectiva argumentación a los planteamientos, en función a las necesidades encontradas en la indagación.

\section{Fuentes Documentales}

El análisis correspondiente a las características que predomina en el tema seleccionado, llevan a incluir diferentes fuentes documentales encargadas de darle el respectivo apoyo y en ese sentido cumplir con la valoración de los hechos a fin de generar nuevos criterios que sirven de referencia a otros procesos investigativos. Para (Arias, 2010), las fuentes documentales incorporadas en la investigación documental o bibliográfica, "representa la suma de materiales sistemáticos que son revisados en forma rigurosa y profunda para llegar a un análisis del fenómeno". (p.41). Por lo tanto, se procedió a cumplir con la realización de una lectura previa determinada por encontrar aquellos aspectos estrechamente vinculados con el Análisis de "Patologías Específicas de importancia en la UCI”, para luego explicar mediante un desarrollo las respectivas apreciaciones generales de importancia.

\section{Técnicas para la Recolección de la Información}

La conducción de la investigación para ser realizada en función a las particularidades que determinan a los estudios documentales, tiene como fin el desarrollo de un conjunto de acciones encargadas de llevar a la selección de técnicas estrechamente vinculadas con las características del estudio. En tal sentido, (Arias Obcit) refiere, que es "una técnica particular para aportar ayuda a los procedimientos de selección de las ideas primarias y secundarias”. (p. 71).

Por ello, se procedió a la utilización del subrayado, resúmenes, fichaje, como parte básica para la revisión y selección de los documentos que presentan el contenido teórico. Es decir, que mediante su aplicación de estas técnicas se pudo llegar a recoger informaciones en cuanto a la revisión bibliográfica de los diversos elementos encargados de orientar el proceso de investigación. Tal como lo expresa, (Arias Obcit) "las técnicas documentales proporcionan las herramientas esenciales y determinantes para responder a los objetivos formulados y llegar a 


\section{Patologías Específicas de Importancia en la U.C.I}

Vol. 3, núm. 2., (2019)

Jean Carlos Delgado Macías; Vilma Geovanna Lara Fajardo; Luiz Carlos Flores Tacle; Bairon

Andrés Sabando Farías; Edwin Gabriel Aguilar Sánchez; Glenda Aracely Fernández Zambrano

resultados efectivos" (p. 58). Es decir, para responder con eficiencia a las necesidades investigativas, se introdujeron como técnica de recolección el método inductivo, que hizo posible llevar a cabo una valoración de los hechos de forma particular para llegar a la explicación desde una visión general.

Asimismo, se emplearon las técnicas de análisis de información para la realización de la investigación que fue ejecutada bajo la dinámica de aplicar diversos elementos encargados de determinar el camino a recorrer por el estudio, según, (Arias, Obcit) las técnicas de procesamiento de datos en los estudios documentales "son las encargadas de ofrecer al investigador la visión o pasos que debe cumplir durante su ejercicio, cada una de ellas debe estar en correspondencia con el nivel a emplear" (p. 123). Esto indica, que para llevar a cabo el procesamiento de los datos obtenidos, es necesario establecer las técnicas que serán seleccionadas, destacándose en este caso, de manera particular: fichas de resumen, textual, registros descriptivos entre otros, los mismos se deben ajustar al nivel que ha sido seleccionado.

\section{Resultados.}

La Unidad de cuidados Intensivos UCI, representa un área asistencial dotada de la tecnología médica más avanzada y especialistas intensivistas encargados de brindar los cuidados médicos necesarios a aquellos pacientes que se encuentran en condiciones críticas de salud. Entre las patologías más recurrentes se encuentran: 


\section{Patologías Específicas de Importancia en la U.C.I}

Vol. 3, núm. 2., (2019)

Jean Carlos Delgado Macías; Vilma Geovanna Lara Fajardo; Luiz Carlos Flores Tacle; Bairon Andrés Sabando Farías; Edwin Gabriel Aguilar Sánchez; Glenda Aracely Fernández Zambrano

Tabla $\mathbf{N}^{\circ}$ 1. Diagnósticos más recurrentes de ingreso a la UCI

\begin{tabular}{|l|c|c|}
\hline Diagnósticos & Frecuencia & Porcentaje \\
\hline Choque séptico & 35 & 13.83 \\
Síndrome coronario agudo & 35 & 13.83 \\
Estado posoperatorio & 32 & 12.64 \\
Enfermedad neurológica & 26 & 10.27 \\
Patología gastrointestinal & 22 & 8.69 \\
Sepsis pulmonar & 19 & 7.50 \\
Falla ventilatoria no infecciosa & 14 & 5.53 \\
Trauma craneoencefálico & 12 & 4.74 \\
Arritmia & 11 & 4.34 \\
Trauma & 9 & 3.55 \\
Ataque cerebrovascular & 8 & 3.16 \\
Neoplasia & 7 & 2.76 \\
Posoperatorio revascularización miocárdica & 6 & 2.37 \\
Edema pulmonar cardiogénico & 6 & 2.37 \\
Cetoacidosis diabética & 5 & 1.97 \\
Otros & 6 & 2.37 \\
\hline Total & 253 & 100 \\
\hline
\end{tabular}

Fuente:(Almao, 2016).

1.- Insuficiencia Respiratoria Representa otra de las principales causas de ingreso en UCI, y requiere generalmente la conexión al respirador. En ocasiones, el inicio de los síntomas coincide con una enfermedad sistémica (especialmente hipotiroidismo o hipertiroidismo), con infecciones intercurrentes, fiebre, agotamiento físico o emocional y embarazo o puerperio. En caso contrario, se trata de una insuficiencia respiratoria con afección de pares craneales, por lo que se debe establecer el diagnóstico diferencial con el botulismo, en el que habrá antecedentes epidemiológicos y de cuadro gastrointestinal, polimiositis, esclerosis lateral amioatrófica y síndrome miasteniforme de Eaton-Lambert. (Cols 2016). 


\section{Patologías Específicas de Importancia en la U.C.I}

Vol. 3, núm. 2., (2019)

Jean Carlos Delgado Macías; Vilma Geovanna Lara Fajardo; Luiz Carlos Flores Tacle; Bairon

Andrés Sabando Farías; Edwin Gabriel Aguilar Sánchez; Glenda Aracely Fernández Zambrano

2.- Hemorragia digestiva alta no varicosa: Esta patología se encuentra asociada a una mortalidad entre un $5 \%$ en pacientes ambulatorios y un $33 \%$ en pacientes hospitalizados. Lo más importante y prioritario ante la existencia de una hemorragia digestiva alta es valorar el estado hemodinámico del paciente, realizando una estratificación inicial del riesgo preendoscópico. En estos casos siempre se debe ingresar al paciente en una Unidad de cuidados intensivos para estabilización, y posterior realización de endoscopia digestiva alta.

La Hemorragia digestiva grave como hemos mencionado, es aquella en la que hay evidencia de hipovolemia con una frecuencia cardiaca $>100 \mathrm{lpm}$ o TAS $<100 \mathrm{mmHg}$, junto con datos de hipoperfusión periférica. La prioridad en estos casos es la resucitación en una Unidad de cuidados intensivos, restaurando el volumen circulante de forma urgente con cristaloides (suero fisiológico), siendo los objetivos: TAS > $100 \mathrm{mmHg}$, presión venosa central entre 0-5 cm H2O y un débito urinario $>30 \mathrm{ml} /$ hora. Además de unas medidas generales como la oxigenoterapia, reservar 4 concentrados de hematíes, y siempre y de forma precoz consultar con endoscopista de guardia. (OMS 2017).

3.- Pancreatitis Aguda: Representa una causa frecuente de ingreso a una unidad de cuidados intensivos, esta patología corresponde a una enfermedad cuya primera causa es de origen biliar, esto se debe a cálculos que migran desde la vesícula a los conductos biliares. Si se ocluye cualquiera de estos conductos por un cálculo, puede inflamarse el páncreas y generar una intensa respuesta inflamatoria que es variable y que en algunos casos se comporta como una Pancreatitis Aguda Grave, la que puede asociarse a un elevado riesgo de fallecer, o bien a estadías prolongadas en UCI.

Esta enfermedad se encuentra asociada a una mortalidad global entre un 2-5\% y en las formas graves hasta un 50\%. Entre un 15-25\% de los pacientes con pancreatitis aguda desarrollarán una pancreatitis aguda severa, que se asocia a una alta tasa de complicaciones, entre ellas el SRIS (resultado de la inflamación intra y extrapancreática), y representa casi la mitad de la mortalidad por pancreatitis severa. 


\section{Patologías Específicas de Importancia en la U.C.I}

Vol. 3, núm. 2., (2019)

Jean Carlos Delgado Macías; Vilma Geovanna Lara Fajardo; Luiz Carlos Flores Tacle; Bairon Andrés Sabando Farías; Edwin Gabriel Aguilar Sánchez; Glenda Aracely Fernández Zambrano

Los criterios de gravedad se basan en:

1. Parámetros analíticos: Leucocitosis $>16.000$, glucemia $>200$ en pacientes no diabéticos, uremia $>60$, GOT $>250, \mathrm{LDH}>600$, descenso del hematocrito $>10$ puntos, calcio $<8$ $\mathrm{mg} / \mathrm{dl}, \mathrm{PaO} 2<60 \mathrm{mmHg}$, exceso de bases -4 , aumento de PCR.

2. TAC: Áreas necrótico/hemorrágicas.

3. Punción-lavado peritoneal: Líquido libre de color oscuro, líquido libre $>20 \mathrm{ml}$, líquido de lavado de tonalidad más intensa que la fresa.

4. Circunstancias que por sí solas confieren gravedad: Masa palpable, hemorragia digestiva alta, hemoperitoneo, disnea del adulto, coagulación intravascular diseminada (CID).

Cuando se tienen 3 o más de los criterios clínico-analíticos en las primeras 48 horas del ingreso, un lavado peritoneal o un TAC con áreas de necrosis pancreática extensa (>50\%) definen una pancreatitis como grave y deben ingresar en una UCI con los siguientes objetivos: Reposo pancreático, alivio del dolor, estabilización hemodinámica, correcciones metabólicas y equilibrio ácido-base, prevención de hemorragia digestiva y procesos infecciosos. (Cols 2016).

4.-Fallo Hepático Agudo: Se define como una rápida evolución de una lesión hepática aguda y grave con deterioro de la función sintética y encefalopatía en una persona con un hígado previamente normal, o con enfermedad hepática compensada. Todos los pacientes con un fallo hepático agudo fulminante deben estar ingresados en una unidad de cuidados intensivos UCI y valorar la posibilidad de un trasplante hepático, ya que la mortalidad asociada es alta y es el único tratamiento eficaz con una supervivencia al año > al $80 \%$.

Como complicaciones graves asociadas a esta enfermedad se tiene, desde la propia encefalopatía que forma parte de la definición hasta edema cerebral, fallo renal, hipoglucemia, acidosis metabólica, sepsis, coagulo Patía y fallo multi - orgánico.(Arreaza, 2015).

5.- Lesiones Esofagogástricas por Caústicos: Se asocian una mortalidad global del 1-3\%. La gravedad va a depender del tipo de caústico, concentración del tóxico, estado de replección 


\section{Patologías Específicas de Importancia en la U.C.I}

Vol. 3, núm. 2., (2019)

Jean Carlos Delgado Macías; Vilma Geovanna Lara Fajardo; Luiz Carlos Flores Tacle; Bairon

Andrés Sabando Farías; Edwin Gabriel Aguilar Sánchez; Glenda Aracely Fernández Zambrano

gástrica, tiempo de contacto con la mucosa. Ante esto lo prioritario siempre es asegurar la vía aérea, estabilizar la situación hemodinámica, mantener al paciente en ayunas, y evitar algunos errores frecuentes como son:

- Provocar el vómito, ya que produce una reexposición al caústico.

- Neutralizar el agente, produce una reacción exotérmica y aumenta el daño.

- Colocación de SNG, se relaciona con una mayor tasa de vómitos y riesgo de perforación.

En todos los casos de debe obtener analítica, gases venosos, radiografía simple de tórax y abdomen, y para descartar posible perforación visceral la técnica de elección es el TAC con contraste intravenoso. Si aparecen complicaciones de riesgo vital como mediastinitis, peritonitis, distrés o estado crítico (inestabilidad hemodinámica, disnea o estridor respiratorio), el paciente debe ser trasladado de forma urgente a una Unidad de Cuidados Intensivos UCI, y tras la estabilización del mismo, y descartada la perforación, se realizará una endoscopia para cuantificación de los daños, debiendo hacerse ésta en las primeras 24-48 horas. La clasificación de ZARGAR tiene valor pronóstico y se obtiene por endoscopia. (Lint, 2016).

6.- Perforación de Esófago: Complicación altamente letal, y la más grave de todo el tracto gastrointestinal. Requiere en la mayor parte de los casos una intervención quirúrgica inmediata. Estos pacientes siempre deben ingresarse, y preferentemente en una Unidad de cuidados intensivos UCI. Se debe prestar especial atención al compromiso de la vía aérea, y al posible desarrollo de shock. Los síntomas fundamentales son el dolor cervical o torácico, la disfagia, ronquera, afonía, disnea o sepsis, y se manifiesta en forma de fiebre, taquicardia o leucocitosis.

Los signos más frecuentes son la crepitación subcutánea, el signo de Hamman (Ruido de crujido o chasquido que se oye sincrónico con los latidos del corazón, sobre el precordio y mejor en decúbito lateral, debido a la presencia de aire en los tejidos), la fiebre y el shock. Se debe actuar con cautela en estos pacientes porque cualquiera de estos síntomas puede estar ausente durante las primeras horas, y su ausencia no descarta el diagnóstico 


\section{Patologías Específicas de Importancia en la U.C.I}

Vol. 3, núm. 2., (2019)

Jean Carlos Delgado Macías; Vilma Geovanna Lara Fajardo; Luiz Carlos Flores Tacle; Bairon Andrés Sabando Farías; Edwin Gabriel Aguilar Sánchez; Glenda Aracely Fernández Zambrano

7.- Colitis Fulminante: Es una inflamación grave del colon asociada a toxicidad sistémica, con o sin dilatación colónica asociada. Los pacientes se tratarán con antibioticoterapia de amplio espectro, corticoides intravenosos, analgesia y si no responden habrá que valorar el uso de infliximab / ciclosporina, o cirugía. La causa más común es la enfermedad inflamatoria intestinal (colitis ulcerosa y enfermedad de Crohn), pero también se presenta en cuadros infecciosos severos del colon (clostridiumdifficile, campylobacter, salmonella, entamoebahystolitica), por falta de oxigenación de las células del colon (isquemia), cuando hay vólvulos o divertículos en colon, o por obstrucción del colon por cáncer.

Clínicamente se presentarán con: Fiebre alta, taquicardia, leucocitosis, anemia, distensión/dolor abdominal, pérdida de peso, hemorragia que requiere trasfusión, y alteraciones hidroelectrolíticas. Deben Ingresarse en una Unidad de Cuidados Intensivos UCI, por el alto riesgo de megacolon tóxico, perforación grave o hemorragia grave. (Lint 2016).

8.- Megacolon Tóxico: Complicación grave que se caracteriza por una dilatación aguda del intestino grueso, mayor a $6 \mathrm{~cm}$, a nivel de colon transverso, de forma segmentaria o total, no obstructiva, acompañada de signos de toxicidad sistémica. Debe sospecharse ante la asociación de colitis grave junto con dilatación colónica y signos de toxicidad (fiebre, taquicardia, anemia y aumento de VSG). Si aparece aire libre intraperitoneal, intramural o en vena porta, pensar en que el paciente asocia una perforación.

Los criterios diagnósticos actualmente admitidos son:

- Evidencia radiológica de dilatación colónica.

- Tres o más de los siguientes: Fiebre $>38^{\circ} \mathrm{C}$, frecuencia cardiaca $>120 \mathrm{lpm}$, leucocitosis neutrofílica> 10.5 x 10.9 .

- Anemia con $\mathrm{Hb}$ o hematocrito $<60 \%$ de las cifras normales.

- Además de lo anterior, como mínimo uno de los siguientes: Deshidratación, alteración del estado de conciencia, alteración electrolitos, hipotensión. 


\section{Patologías Específicas de Importancia en la U.C.I}

Vol. 3, núm. 2., (2019)

Jean Carlos Delgado Macías; Vilma Geovanna Lara Fajardo; Luiz Carlos Flores Tacle; Bairon

Andrés Sabando Farías; Edwin Gabriel Aguilar Sánchez; Glenda Aracely Fernández Zambrano

Analíticamente como signo de mal pronóstico se va a encontrar una alcalosis metabólica, y el tratamiento siempre irá dirigido a controlar la causa desencadenante y reducir la dilatación colónica y preferentemente en una Unidad de Cuidados Intensivos UCI. (Lint 2016).

9.- Gastroenteritis Aguda: Se define como todo proceso diarreico de menos de 2 semanas de evolución, que se puede acompañar de náuseas, vómitos y dolor abdominal. Existen dos tipos:

- Gastroenteritis aguda no inflamatoria - Cursa con hemoconcentración, insuficiencia renal por deshidratación, hipernatremia, hipopotasemia y acidosis metabólica en grado variable.

- Gastroenteritis aguda inflamatoria - Cuya complicación más importante es la sepsis.

Los criterios de ingreso en UCI en estos pacientes son:

- Intolerancia oral, o importante número de deposiciones que causen deshidratación o alteraciones hidroelectrolíticas.

- Inestabilidad hemodinámica (criterios de Shock).

- Afección de base descompensada por el proceso infeccioso aguda gastrointestinal o > 65 años con diarrea inflamatoria.

- Gastroenteritis agudas con riesgo vital como son el botulismo o el cólera, en las que se puede o bien afectar la musculatura respiratoria o causar intensa deshidratación. (Lint 2016).

10.- Dolor abdominal agudo: Representa un auténtico desafío para el médico, o principal ante un paciente con esta clínica es plantearse 3 cuestiones:

1. ¿Necesita tratamiento quirúrgico o puede manejarse de forma ambulatoria?

2. ¿Cuál es el diagnóstico más probable? 


\section{Patologías Específicas de Importancia en la U.C.I}

Vol. 3, núm. 2., (2019)

Jean Carlos Delgado Macías; Vilma Geovanna Lara Fajardo; Luiz Carlos Flores Tacle; Bairon Andrés Sabando Farías; Edwin Gabriel Aguilar Sánchez; Glenda Aracely Fernández Zambrano

3. ¿Entraña un riesgo vital para el enfermo?, entre estas podemos destacar la rotura de esófago, la perforación de víscera hueca, la obstrucción intestinal con estrangulación, la pancreatitis necrotizante, la hemorragia intraabdominal, y la isquemia mesentérica.

Hay que prestar una especial atención a tres factores que inducen a una mayor incidencia de errores, como son el sexo femenino, la edad avanzada, y lo estados de inmunosupresión. Los criterios de ingreso en una Unidad de cuidados Intensivos UCI son el deterioro clínico rápidamente progresivo con neumoperitoneo, peritonitis difusa aguda o inestabilidad hemodinámica incontrolable y probablemente, en estos casos serán candidatos a una cirugía urgente.(Berro, 2014).

11.- La Patología Neuromuscular en los pacientes críticos ha comenzado a ser objeto de un importante número de estudios en los últimos años, si bien aún quedan muchas lagunas en el conocimiento de su etiología, patogenia, tratamiento y pronóstico. Dentro de esta patología debemos distinguir dos grandes grupos. En el primero, la debilidad muscular aparece antes del ingreso en UCI y es posible identificar una causa conocida. El síndrome de Guillain-Barré y la miastenia grave son las dos entidades que con mayor frecuencia requieren ser atendidas en nuestras unidades. (Millán, 2016).

En el segundo grupo, la debilidad muscular se adquiere en la UCI, en pacientes sin enfermedad neuromuscular previa, y es secundaria a la gravedad de la enfermedad que originó su ingreso en esta unidad y/o al tratamiento empleado. La polineuropatía del paciente crítico (PPC) es, de todas ellas, la entidad más precisamente definida y de la que conocemos mejor sus características clínicas, diagnóstico y pronóstico; no obstante, aún quedan muchas sombras en cuanto a su etiopatogenia. 


\section{Patologías Específicas de Importancia en la U.C.I}

Vol. 3, núm. 2., (2019)

Jean Carlos Delgado Macías; Vilma Geovanna Lara Fajardo; Luiz Carlos Flores Tacle; Bairon Andrés Sabando Farías; Edwin Gabriel Aguilar Sánchez; Glenda Aracely Fernández Zambrano

Tabla $\mathbf{N}^{\circ}$ 1. Trastornos neuromusculares en paciente críticos que deben ser ingresados en UCI
Lesión de la médula espinal
Lesión traumática
Compresión epidural (neoplasia, hematoma...)
Mielopatía isquémica
Mielitis transversa aguda
Esclerosis lateral amiotrófica
Trastornos del nervio periférico
Síndrome de Guillain-Barré
Polineuropatía vasculítica
Polineuropatía diabética
Porfiria
Polineuropatía urémica
VIH
Neuropatía nutricional (tiamina, vitamina E)
Polineuropatía relacionada con medicación*
Intoxicación por talio
Trastornos de la sinapsis neuromuscular
Miastenia grave
Intoxicación por organofosforados
Botulismo
Parálisis por mordedura de garrapata
Síndrome de Eaton-Lambert
Toxicidad por aminoglucósidos
Toxicidad por colistina
Hipermagnesemia

\section{Trastornos del músculo}
Distrofia muscular
Poliomiositis
Miopatía mitocondrial

Fuente: (Millán 2016).

Las alteraciones de la placa neuromuscular y sobre todo la miopatía, que frecuentemente coexiste con la PPC, son las otras complicaciones del sistema nervioso periférico que se desarrollan en pacientes críticos. Los avances en el conocimiento de estas afecciones podrían tener un 


\section{Patologías Específicas de Importancia en la U.C.I}

Vol. 3, núm. 2., (2019)

Jean Carlos Delgado Macías; Vilma Geovanna Lara Fajardo; Luiz Carlos Flores Tacle; Bairon Andrés Sabando Farías; Edwin Gabriel Aguilar Sánchez; Glenda Aracely Fernández Zambrano

importante impacto, sobre todo para el desarrollo de intervenciones terapéuticas y preventivas efectivas que mejoren el pronóstico de estos pacientes.

Tabla $\mathbf{N}^{\circ}$ 2. Disfunción del sistema nervioso autónomo en el Síndrome de Guillain - Barré

1. Hiperactividad simpática: hipertensión (mantenida o paroxística a veces seguida de hipotensión), taquicardia sinusal, taquiarritmias supraventriculares

2. Hipoactividad simpática: hipotensión, anhidrosis

3. Disfunción parasimpática: bradiarritmias, asistolia

4. Disfunción intestinal y de vejiga: retención urinaria, estreñimiento, incontinencia

5. Alteraciones del tono vasomotor: estasis venosa, rubor facial

6. Midriasis, hipersalivación, anhidrosis o sudoración excesiva

Fuente: (Millán 2016)

12.- Polineuropatía del paciente Crítico: La polineuropatía del paciente crítico (PPC) es una degeneración axonal primaria de fibras motrices y sensitivas que se acompaña de degeneración de las fibras musculares como resultado de la denervación aguda que sufren dichas fibras del músculo estriado. Ocurre en pacientes críticos, especialmente los que contraen síndrome de respuesta inflamatoria sistémica (SRIS) y sepsis grave con síndrome de disfunción multiorgánica (SDMO). Generalmente, la PPC coexiste con la miopatía del paciente crítico, por lo que en la actualidad muchos autores lo denominan polineuromiopatía del paciente crítico (PNMPC). (Millán 2016).

La forma de presentación es como tetraplejía o tetraparesia, a menudo con dificultad de desconexión del respirador ${ }^{44}$. Los reflejos osteotendinosos suelen estar abolidos, si bien podemos hallarlos reducidos o incluso normales. La concentración sérica de creatincinasa (CK) es normal o está ligeramente elevada. El LCR no presenta alteraciones patológicas 


\section{Patologías Específicas de Importancia en la U.C.I}

Vol. 3, núm. 2., (2019)

Jean Carlos Delgado Macías; Vilma Geovanna Lara Fajardo; Luiz Carlos Flores Tacle; Bairon

Andrés Sabando Farías; Edwin Gabriel Aguilar Sánchez; Glenda Aracely Fernández Zambrano

Figura $N^{\circ}$ 1. Protocolo diagnóstico ante sospecha de enfermedad neuromuscular adquirida en el paciente crítico

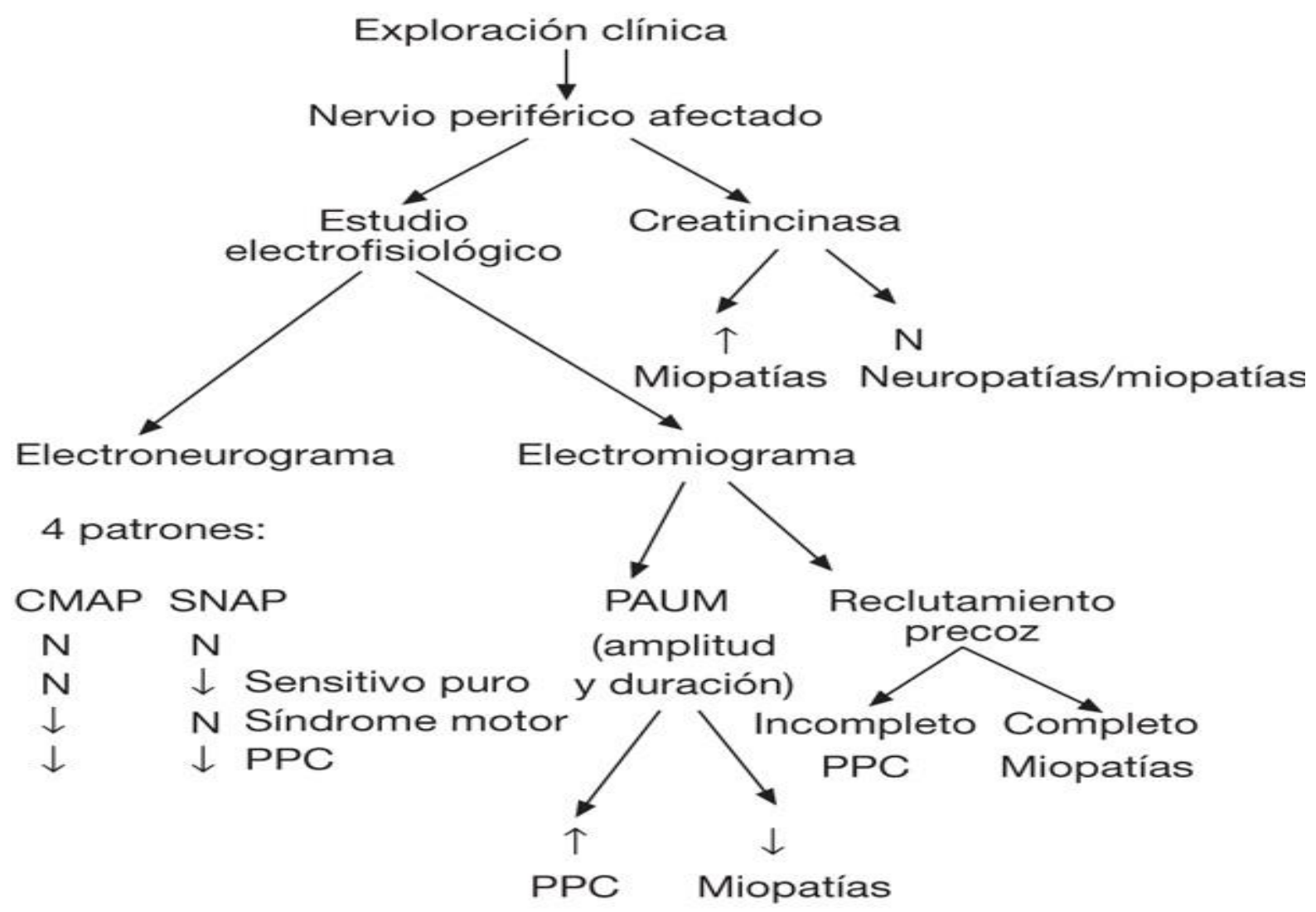

Fuente: (Millán 2016).

En los casos de polineuropatía, la recuperación es favorable en semanas. En los casos de afección grave, el pronóstico funcional no es bueno, y a los 2 años persisten importante limitación de la movilidad y calidad de vida muy deteriorada en casi la totalidad de los pacientes evaluados. La coexistencia de una neuropatía axonal con enlentecimiento de la velocidad de conducción se asocia a una peor recuperación. Una prolongada estancia en UCI, la mayor duración de la sepsis y la pérdida de peso son los tres parámetros que se asocian a una peor recuperación según un estudio reciente que siguió la evolución durante 2 años de 19 pacientes con PP. 


\section{Patologías Específicas de Importancia en la U.C.I}

Vol. 3, núm. 2., (2019)

Jean Carlos Delgado Macías; Vilma Geovanna Lara Fajardo; Luiz Carlos Flores Tacle; Bairon Andrés Sabando Farías; Edwin Gabriel Aguilar Sánchez; Glenda Aracely Fernández Zambrano

13.- Trastornos de la conducción neuromuscular: El bloqueo neuromuscular prolongado tras el uso de pancuronio y vecuronio, especialmente cuando hay insuficiencia hepática o renal, se debe a la acumulación de los 3-hidroxi y 3-desacetil metabolitos que poseen actividad. Diversos trastornos metabólicos tales como hipofosfatemia y en especial la hipermagnesemia pueden exacerbar el bloqueo. El diagnóstico se confirma con la estimulación repetitiva a 3 y $20 \mathrm{~Hz}$ de los nervios periféricos que muestra una reducción progresiva de la transmisión nerviosa. La recuperación ocurre en días o semanas

El cuadro clínico de una miopatía es indistinguible de otras etiologías de enfermedad neuromuscular adquiridas en UCI. Estos pacientes presentan debilidad simétrica de las extremidades y los músculos respiratorios. Los reflejos profundos suelen estar reducidos o ausentes.

Tabla $\mathbf{N}^{\circ}$ 3. Diagnóstico Diferencial de Trastornos Neuromusculares

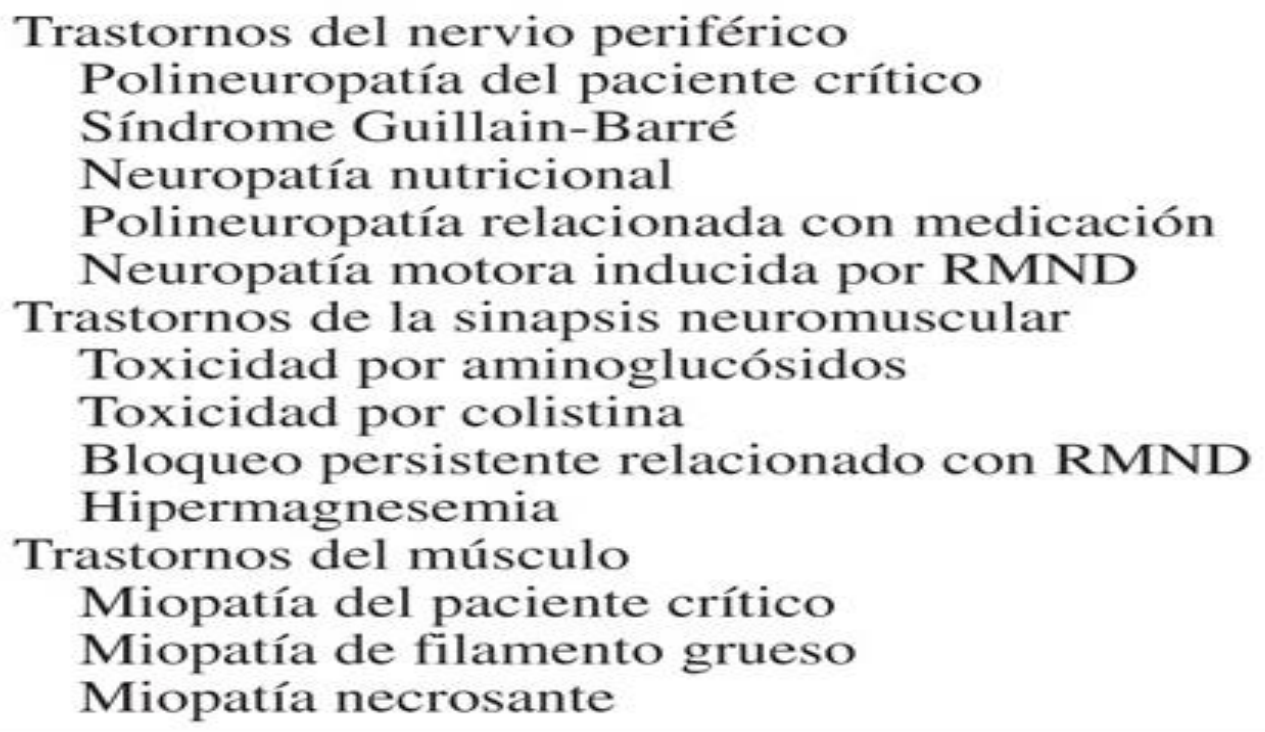

Fuente: (Millán 2016).

14.- Insuficiencia Renal IRA: La IRA se presenta con mayor frecuencia en pacientes hospitalizados, y cerca del 5\% de estos pacientes requieren diálisis. Independiente de su origen, constituye un proceso patológico que encarece ostensiblemente el tratamiento de cualquier persona afectada por este síndrome. La IRA es una complicación común en pacientes 


\section{Patologías Específicas de Importancia en la U.C.I}

Vol. 3, núm. 2., (2019)

Jean Carlos Delgado Macías; Vilma Geovanna Lara Fajardo; Luiz Carlos Flores Tacle; Bairon

Andrés Sabando Farías; Edwin Gabriel Aguilar Sánchez; Glenda Aracely Fernández Zambrano

hospitalizados en la unidad de cuidado intensivo (UCI). Son numerosas las causas asociadas con IRA en pacientes críticos, dentro de ellas la sepsis es la más importante.

La insuficiencia renal se caracteriza por la reducción rápida y progresiva de la función renal. La elevación progresiva de azoados, la cual puede o no acompañarse de oliguria. Esta patología puede presentarse a través de una insuficiencia renal aguda epidemiológica que es común en el 2 a $5 \%$ de los pacientes quirúrgicos, la cual aumenta cuando el $20 \%$ de los pacientes son sometidos a cirugía vascular abdominal. Se clasifica en: Prerenal, Intrínseca y Postrenal.(Campins, 2015).

15.- La sepsis grave, entendida como síndrome, es la primera causa de ingreso a UCI. Se trata de infecciones agudas que producen daño en órganos a distancia del inicialmente enfermo. Por ejemplo, una neumonía grave que produce insuficiencia renal aguda. Representa la principal causa de muerte en los pacientes críticos, con mortalidad promedio entre el 30-50\%. Una de las causas frecuentes de sepsis grave es la neumonía adquirida en la comunidad. Entre el 1-2 \% de las infecciones respiratorias bajas evolucionan hacia la gravedad, con hipotensión (shock) y/o insuficiencia respiratoria, que motiva admisión en UCI y apoyo con ventilador mecánico y/o drogas vasoactivas (medicamentos que suben la presión). La sepsis representa un problema importante en los pacientes hospitalizados en la UCI, porque constituye un factor de riesgo para el desarrollo de Insuficiencia Renal IRA, generando el requerimiento de soporte dialítico y alta mortalidad en esta población

La sepsises la principal causa de muerte en pacientes críticamente enfermos, cerca de 750000 personas al año presentan sepsisy 210000 mueren por esta causa. Actualmente, avances en el conocimiento de su fisiopatología y bases genéticas, han podido cambiar la historia natural de la enfermedad y sus complicaciones como la IRA, causa importante de muerte en estos pacientes. Como se describió, siendo la IRA tan frecuente en UCI sería importante la identificación de los factores de riesgo y el manejo temprano y oportuno de las mismos, lo cual implicaría optimización de recursos, mejoría en la calidad de la atención y disminución de complicaciones y morbimortalidad. En estudios multicéntricos se ha reportado frecuencia entre 39 y $71 \%$ en la población mundial. (Campins 2015). 


\section{Patologías Específicas de Importancia en la U.C.I}

Vol. 3, núm. 2., (2019)

Jean Carlos Delgado Macías; Vilma Geovanna Lara Fajardo; Luiz Carlos Flores Tacle; Bairon Andrés Sabando Farías; Edwin Gabriel Aguilar Sánchez; Glenda Aracely Fernández Zambrano

16- Enfermedades Cerebrovasculares ECV: Las enfermedades cerebrovasculares constituyen, en la actualidad, uno de los más importantes problemas de salud pública. Son la tercera causa de muerte en el mundo, la primera causa de invalidez permanente entre las personas adultas y una de las principales causas de déficit neurológico en el anciano. Por tal motivo La Organización Mundial de la Salud, (OMS), la Asociación Internacional de Hipertensos y otros equipos de expertos insisten en la necesidad de intensificar métodos y estrategias para modificar el estilo de vida pues cada vez es más evidente que para tener cierta efectividad en el tratamiento de la HTA y disminuir las incidencias de enfermedades cerebrovasculares, el paciente debe asumir su responsabilidad y su participación decidida para modificar hábitos hacia conductas más saludables, debido a la repercusión que esta patología tiene en la trayectoria vital del paciente, quien amerita de manera general de ser recluido en la UCI además de ser y representar un elevado costo socio sanitario en el mundo.

El accidente cerebrovascular ECV se presenta cuando la estructura pierde la irrigación sanguínea debido a la interrupción súbita e inmediata del flujo sanguíneo, lo que genera la aparición de una zona infartada y es en ese momento en el cual ocurre el verdadero "infarto cerebral" y se debe sólo a la oclusión de alguna de las arterias que irrigan la masa encefálica, ya sea por acumulación de fibrina, o de calcio o por alguna anormalidad en los eritrocitos, pero generalmente es por ateroesclerosis o bien por un émbolo (embolia cerebral) que procede de otra localización, fundamentalmente el corazón u otras arterias (como la bifurcación de la carótidas o del arco aórtico).(León, 2015).

17.- Enfermedades Cardiovasculares: Las enfermedades cardiovasculares son aquellas que constituyen un conjunto de entidades que afectan el corazón y los vasos sanguíneos. Cuando afecta los vasos sanguíneos puede comprometer órganos como el cerebro (enfermedad cerebrovascular), los miembros inferiores, los riñones y el corazón. Dentro de las enfermedades cardiovasculares las de mayor ocurrencia son la enfermedad coronaria y la enfermedad cerebrovascular. Es por ello, que representa por sí misma una enfermedad, como también un factor de riesgo importante para otras enfermedades, como, por ejemplo, la cardiopatía isquémica, insuficiencia cardiaca, enfermedad cerebro vascular, insuficiencia renal y la Retinopatía hipertensiva. 


\section{Patologías Específicas de Importancia en la U.C.I}

Vol. 3, núm. 2., (2019)

Jean Carlos Delgado Macías; Vilma Geovanna Lara Fajardo; Luiz Carlos Flores Tacle; Bairon

Andrés Sabando Farías; Edwin Gabriel Aguilar Sánchez; Glenda Aracely Fernández Zambrano

Las enfermedades cardiovasculares tales como, ataques al corazón y los accidentes vasculares cerebrales (AVC) suelen ser fenómenos agudos que se deben sobre todo a obstrucciones que impiden que la sangre fluya hacia el corazón o el cerebro. La causa más frecuente es la formación de depósitos de grasa en las paredes de los vasos sanguíneos que irrigan el corazón o el cerebro. De manera general esta patología se debe a hemorragias de los vasos cerebrales o coágulos de sangre que requieren ser atendidas en la UCI. (León 2015)

\section{Conclusiones.}

La UCI, representa un área de servicios asistenciales especializada, que centra su atención en pacientes que presentan un estado crítico de salud. Esta unidad de cuidados representa para el enfermo, un sistema de emergencia que brinda atención especializada a pacientes con riesgos inmediatos de salud, los cuales se deben a un compromiso de las funciones vitales (respiratoria, hemodinámica o neurológica), que, en su efecto, de manera general representan una amenaza potencial para la vida, siendo éste el motivo por el que tal patología debe ser tratada en esta unidad asistencial.

En efecto, las unidades de cuidados intensivos UCI representan un área médica especializada que busca brindar atención personalizada dentro de una asistencia integral, a los lesionados más graves y pacientes con patologías críticas; entiéndase por paciente grave, a aquel que tiene comprometida las funciones de algún órgano, o el que ha sido víctima o sujeto de accidentes generando en ellos lesiones y politraumatismos graves, los cuales les generan riesgos de morir.

Entre las patologías de mayor importancia en la UCI se encuentran las enfermedades Cardiovasculares, Cerebrovasculares, Insuficiencias Renales, Sepsis en estados Críticos, Trastornos Neuromusculares, Colitis Fulminantes, Gastroenteritis Agudas, Perforación del Esófago, Insuficiencias Respiratorias, Hemorragias Digestivas, Fallos Hepáticos, entre otras.

\section{Bibliografía.}

Almao, B. (2016). Patologías de orden Crítico. Revista de Salud de la Habana Cuba, 12.

Arias, F. (2010). Paradigmas de la Investigación Científica. España: Editorial: Luces. 


\section{Patologías Específicas de Importancia en la U.C.I}

Vol. 3, núm. 2., (2019)

Jean Carlos Delgado Macías; Vilma Geovanna Lara Fajardo; Luiz Carlos Flores Tacle; Bairon

Andrés Sabando Farías; Edwin Gabriel Aguilar Sánchez; Glenda Aracely Fernández Zambrano

Arreaza, E. (2015). Coma Seale after brain Trauma. Revista Médica: Journal of neurology, neurosurgery, and psychiatry, 13.

Beoggi, C. (2015). Mortality prediction in head trauma patients: performance of Glasgow Coma Score . Revista de Salud: Critical care medicine,, 22.

Berro, P. (2014). Identificatión of an age cutoff for mortality in patients trauma. Revista: American Journal of Emergency Medicine, 22.

Campins, D. (2015). Crisis de Insufuciencia Renal. Revista Vida y Salud de la Universidad del Zulia LUZ. Venezuela. , 15.

Cols, D. (2016). Estudio epidemiológico autópsico de 784 fallecimientos por traumatismo . Revista de Med Intensiva, de Buenos Aires Arhentina, 25.

Dávila, N. (2012). Paradigmas de la Investigación Científica. Pereire, Colombia: Editorial: Las Brisas.

Hendrick, A. (2017). Incidencia de accidentes bacteriológicos Clinic. Revista: The Journal of trauma. EEUU, 33.

León, M. (2015). Enfermedades Cardiovasculares y sus complicaciones Médicas. Revista Cardiológica de la Universidad de Los Andes, Mérida Venezuela., 9.

Lint, R. (2016). Lethal and time to death in a level trauma. Revista: Journal of the American College of Surgeons, 24.

Millán, G. (2016). A revisión of the trauma score. Revista: he Journal of trauma, 16.

OMS, O. M. (2017). Patologías de mayor incidencia en las Unidades de Cuidados Intensivos UCIs. Revista Médica de la Universidad de la Rioja en Colombia, Medellín. , 14.

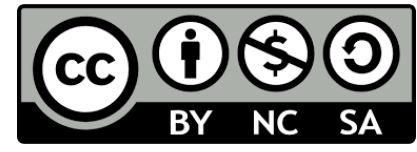

RECONOCIMIENTO-NOCOMERCIAL-COMPARTIRIGUAL

CC BY-NC-SA

ESTA LICENCIA PERMITE A OTROS ENTREMEZCLAR, AJUSTAR Y CONSTRUIR A PARTIR DE SU OBRA CON FINES NO COMERCIALES, SIEMPRE Y CUANDO LE RECONOZCAN LA AUTORÍA Y SUS NUEVAS CREACIONES ESTÉN BAJO UNA LICENCIA CON LOS MISMOS TÉRMINOS. 